Supporting Information

\title{
Dynamic Regulation of Lithium Dendrite Growth with Electromechanical Coupling Effect of Soft $\mathrm{BaTiO}_{3}$ Ceramic Nanofiber Films
}

Shuhui Xia†, Yun Zhao †, Jianhua Yan†, $\neq^{*}$, Jianyong Yuł and Bin Ding $\neq^{*}$

$\dagger$ Key Laboratory of Textile Science \& Technology, College of Textile, Donghua University, Shanghai 201620, China

\#Innovation Center for Textile Science and Technology, Donghua University, Shanghai 200051, China

Corresponding authors:Jianhua Yan, yanjianhua@dhu.edu.cn

Bin Ding, binding@dhu.edu.cn 


\section{Supplementary Figures}

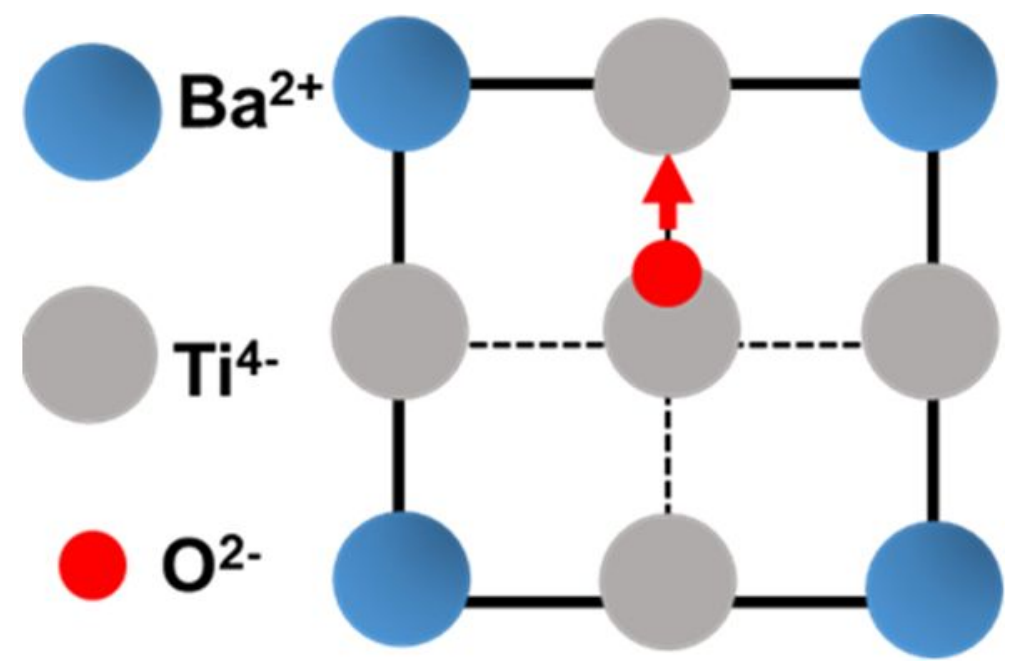

Figure S1. The schematic figrue of the crystal structure of BTO and its spontaneous polarization. As a classic perovskite structural material, the center of the oxygen octahedron of $\mathrm{BTO}$ is a $\mathrm{Ti}^{4+}$ with a small radius, and $\mathrm{O}^{2-}$ is squeezed in between $\mathrm{Ba}^{2+}$ with a large radius. The centers of $\mathrm{O}^{2-}$ and $\mathrm{Ti}^{4+}$ in the unit cell are no longer coincided under the action of the highvoltage electric field, and polarization from negative charges to positive charges is spontaneous generated. 


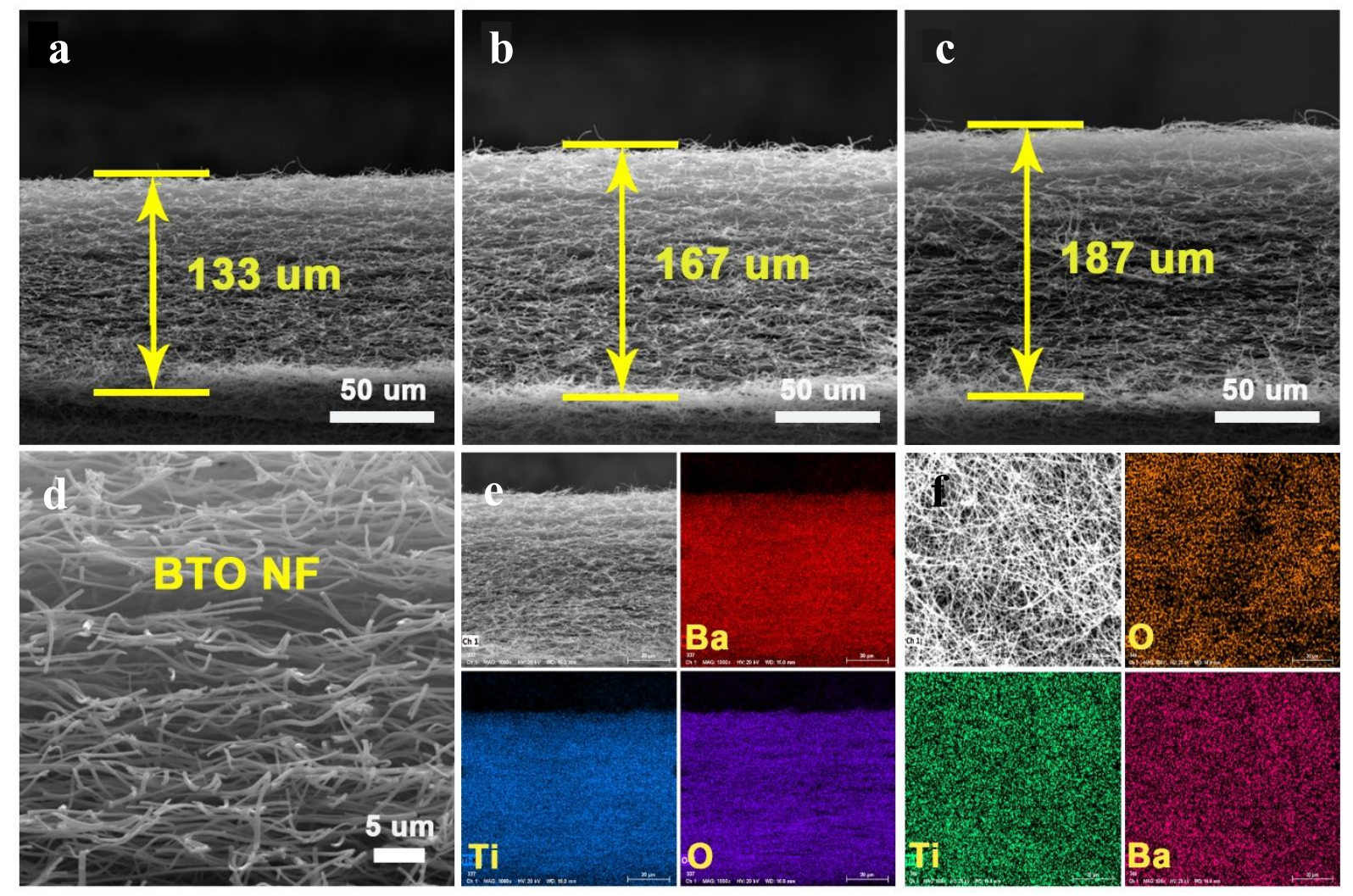

Figure S2. (a-d) The cross-sectional images of the electrospun BTO NF films with different thicknesses, which could be accurately controlled by changing the electrospinning time. The individual NFs in the film entangled with each other and created a high degree of interconnectivity. (e) The cross-sectional and (f) top-view EDS mappings of a BTO NF film, in which $\mathrm{Ba}$, Ti and $\mathrm{O}$ were evenly distributed throughout the film. 


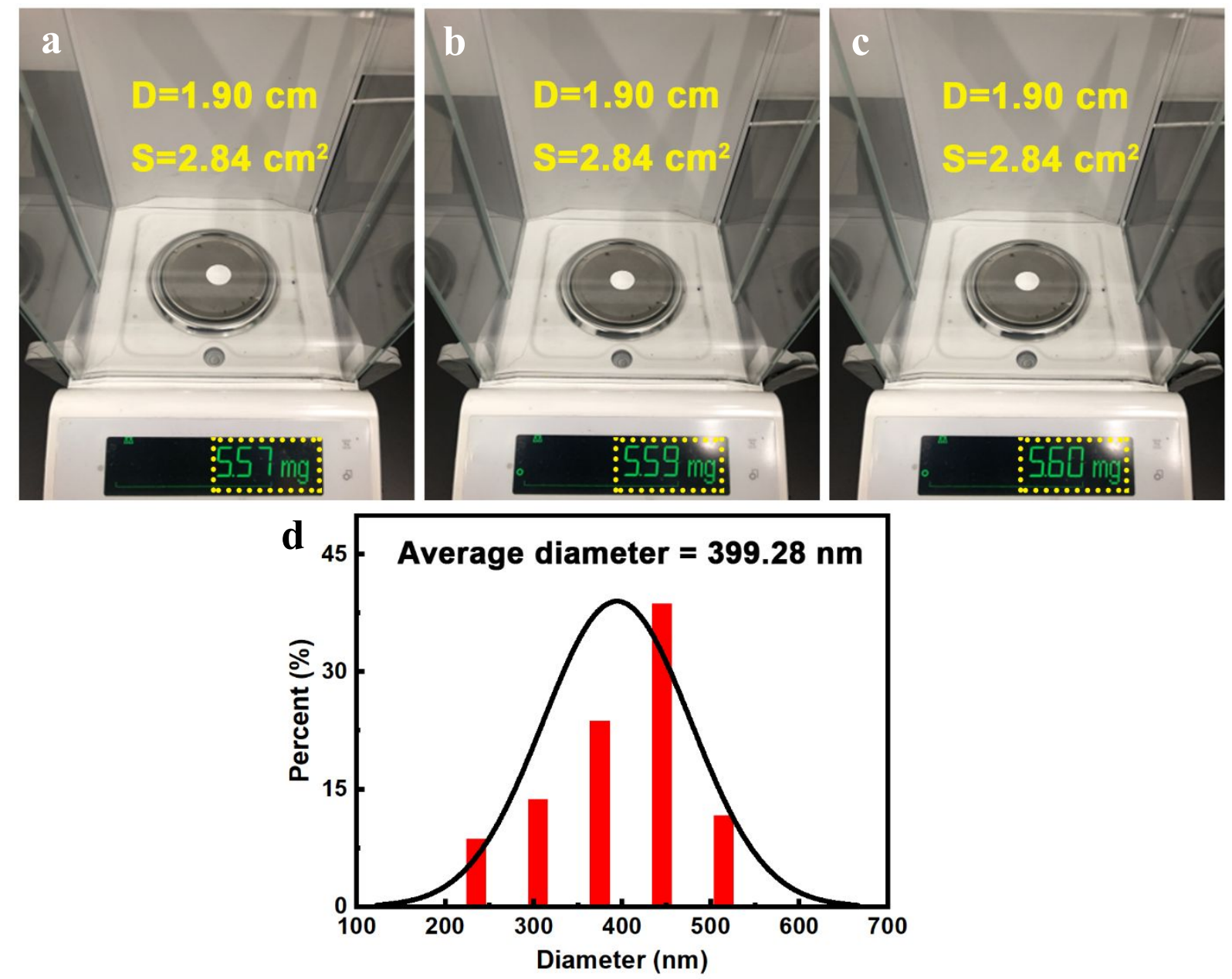

Figure S3. Physical properties of the BTO NF film. (a-c) The weights of the BTO NF films. The diameter of the circular BTO film was $1.90 \mathrm{~cm}$, and the corresponding area was $2.84 \mathrm{~cm}^{2}$. When the thickness was $87.5 \mu \mathrm{m}$, the average mass was measured as $1.96 \mathrm{mg} / \mathrm{cm}^{2}$. (d) The diameter histograms of BTO NFs. The average diameters were calculated as $399.28 \mathrm{~nm}$. 

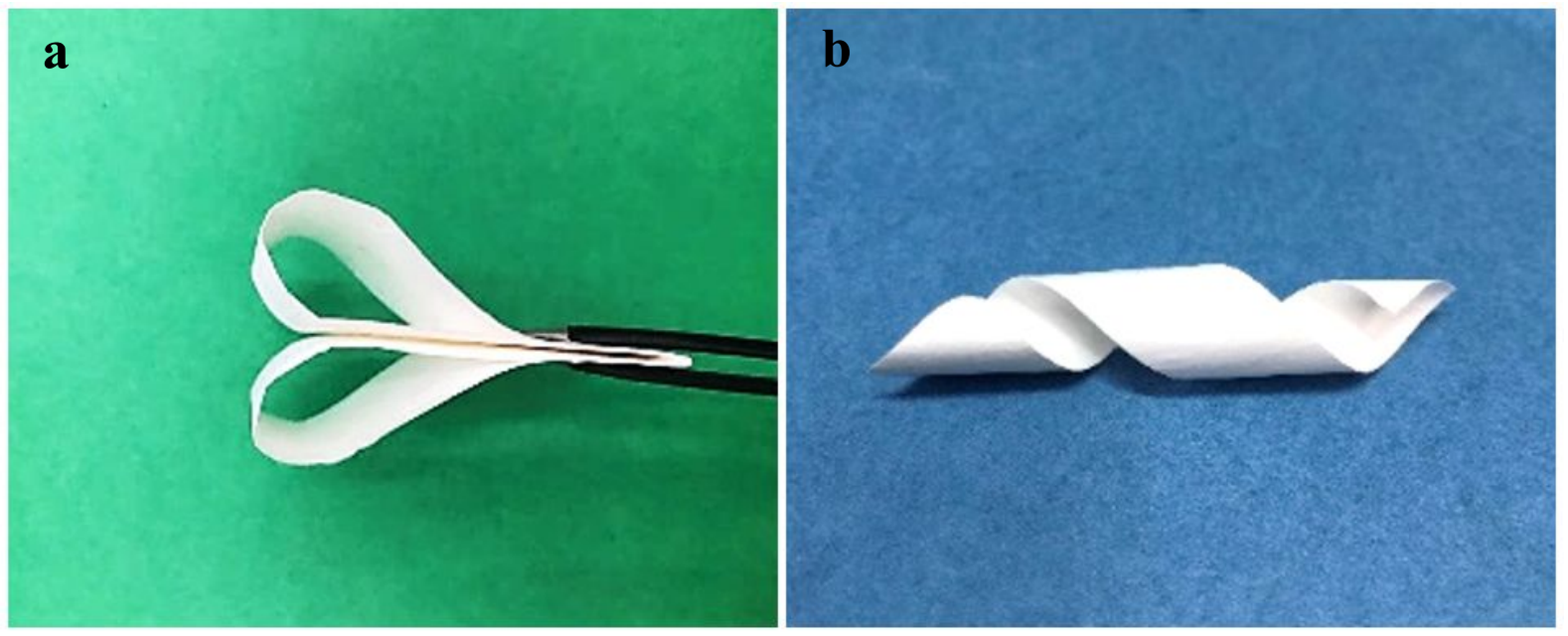

Figure S4. Flexure demonstration of the soft BTO NF films. After being deformed by bending, folding or winding, the BTO NF films could still maintain their original shapes without creating cracks, exhibiting superior flexibility and excellent mechanical properties. 

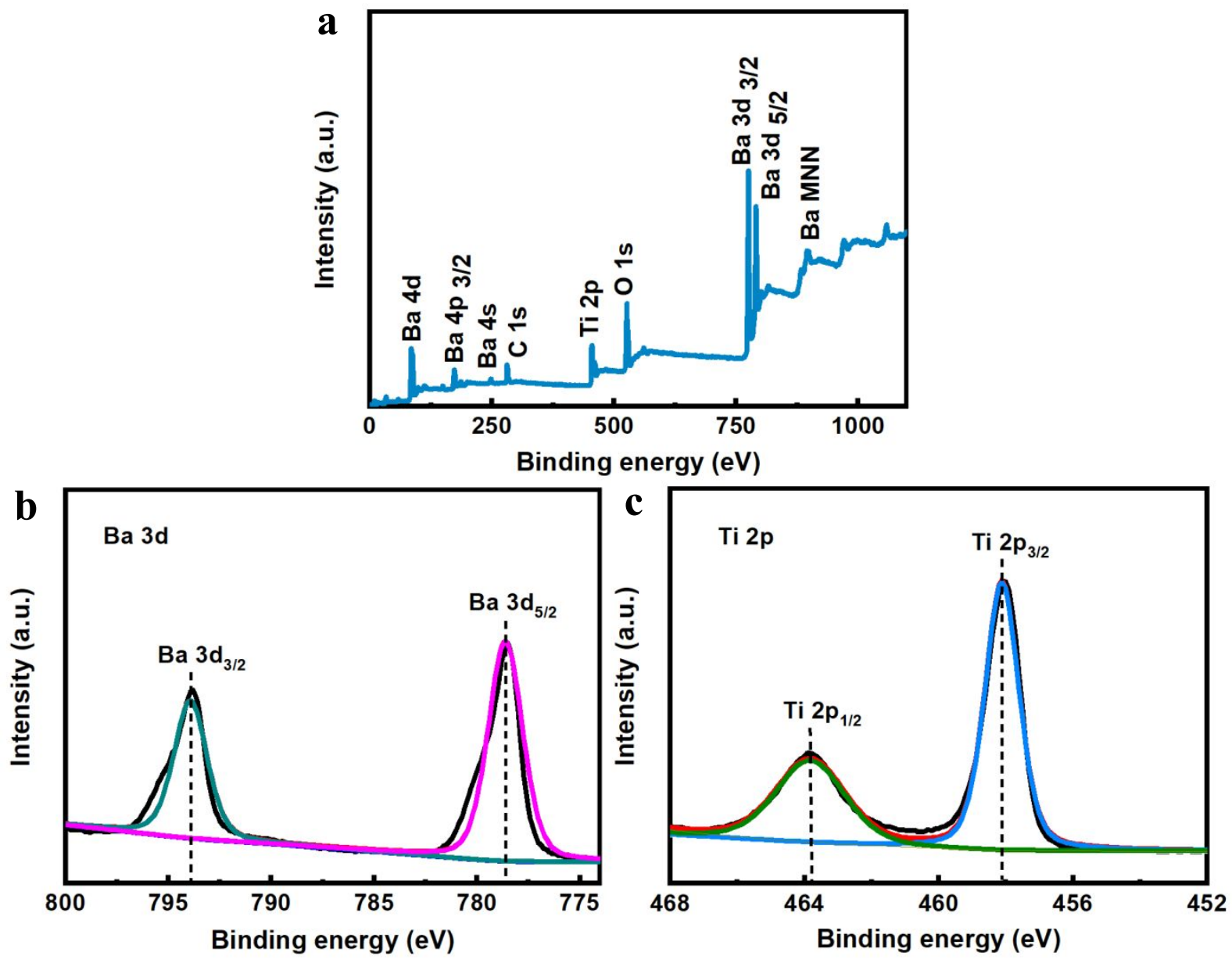

Figure S5. XPS spectra of the soft BTO NFs. From the high-resolution spectrum of Ti $2 p$, the characteristic peaks of Ti $2 \mathrm{p}_{1 / 2}$ and $\mathrm{Ti} 2 \mathrm{p}_{3 / 2}$ appeared at $463.8 \mathrm{eV}$ and $453.0 \mathrm{eV}$ corresponded to the $\mathrm{Ti}^{4+}$ species. The characteristic peaks of $\mathrm{Ba} 3 \mathrm{~d}_{5 / 2}$ and $\mathrm{Ba} 3 \mathrm{~d}_{3 / 2}$ appeared at $778.6 \mathrm{eV}$ and $793.8 \mathrm{eV}$ form the high-resolution spectrum of $\mathrm{Ba} 3 \mathrm{~d}$ corresponded to the $\mathrm{Ba}^{2+}$ species. Therefore, there were not other phase in the BTO NFs. 

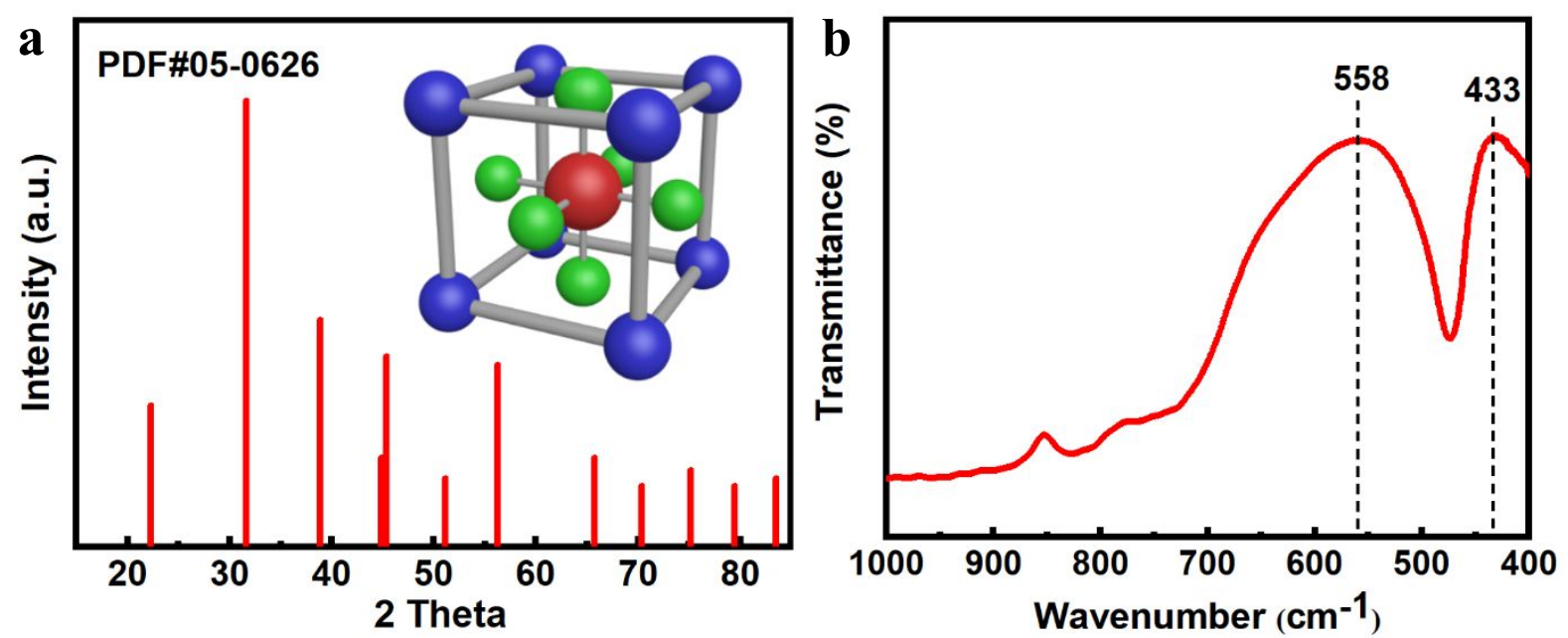

Figure S6. Verification of tetragonal crystal structures of BTO NFs. (a) XRD patterns of standard BTO crystals (PDF\#05-0626). (b) FTIR spectrum of the BTO NF films within 400 $\mathrm{cm}^{-1}$ to $1000 \mathrm{~cm}^{-1}$. Ferroelectricity in perovskite ferroelectric materials depends on the vibration of crystal lattices. The FTIR spectrum provided effective information to study the relationship between the physical properties and microstructures in ferroelectric materials. The two strong absorption peaks appeared at $558 \mathrm{~cm}^{-1}$ and $433 \mathrm{~cm}^{-1}$ corresponded to Ti-O $\mathrm{O}_{\text {I }}^{\text {"stretching" normal }}$ vibration and $\mathrm{Ti}-\mathrm{O}_{\mathrm{II}}$ "bending" normal vibration of Ti-O bond, respectively. Among them, the $\mathrm{Ti}-\mathrm{O}_{\mathrm{I}}$ "stretching" normal vibration of Ti-O bond was very important, because the direction of "stretching" normal vibration was along with that of spontaneous polarization in BTO with tetragonal phase, which indicated that the nanofiber films has a tetragonal BTO crystal structure with spontaneous polarization. 
a

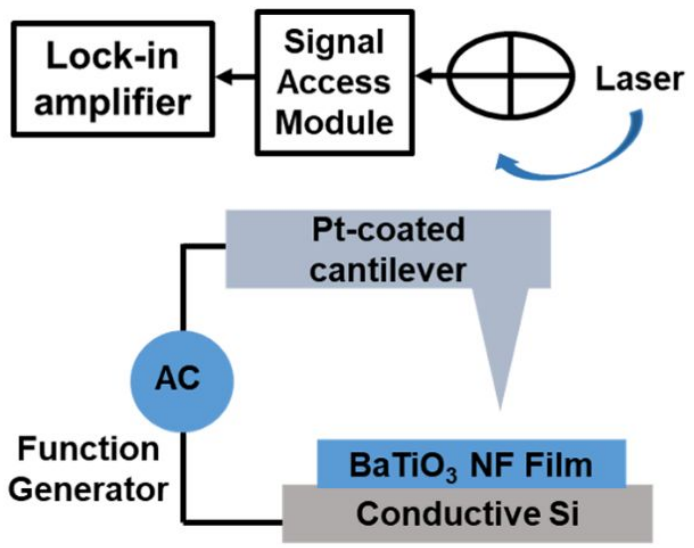

Schematic of the PFM setup

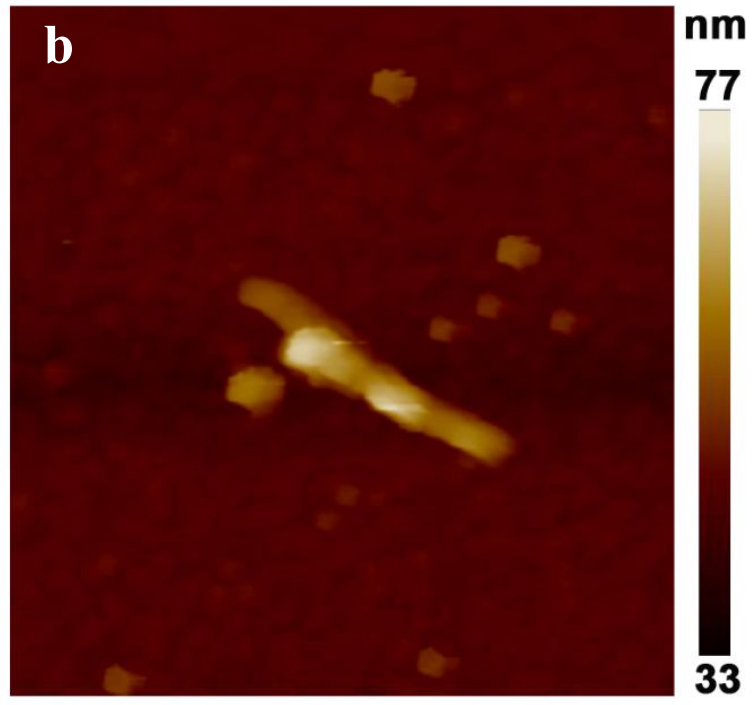

33

Figure S7. PFM tests of BTO NFs. (a) A schematic diagram of the working principle of PFM.

(b) The measured morphology image, in which BTO NFs could be clearly detected. 

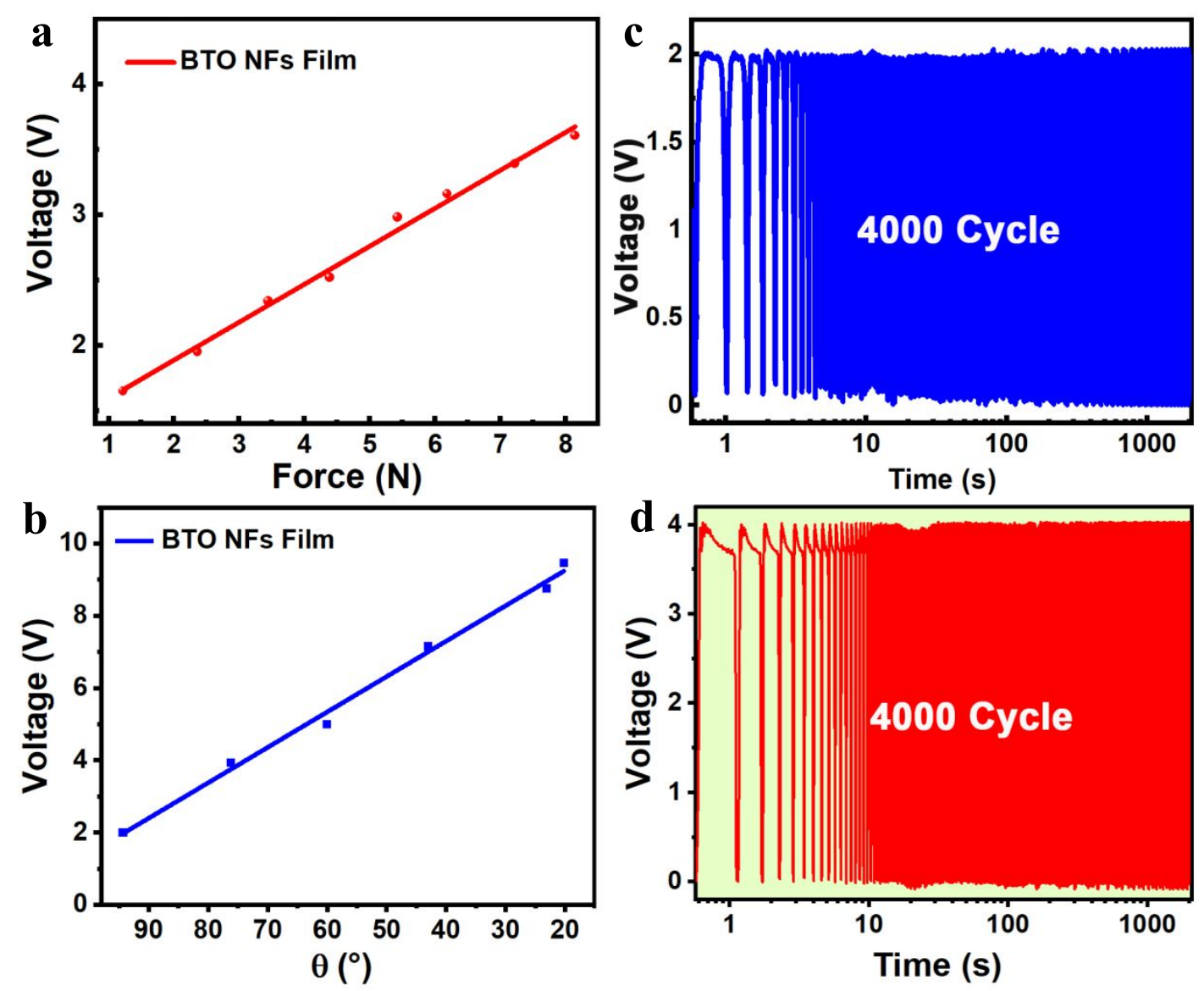

Figure S8. Electrical characteristics of sensor patches that constructed from the soft BTO

NF films. The voltage of sensor patches depended on (a) different force under pressing mode and (b) different bending angles under bending mode. The voltage was increased with the compressive force under the pressing mode, but it was increased when decreasing the bending angle under the bending mode. This was because the smaller the bending angles, the greater the compression deformation of the BTO films, which in turn affected the electrical properties. The sensor patch had voltage sensitivities of $29 \mathrm{mV} \cdot \mathrm{Pa}^{-1}$ and $98.0 \mathrm{mV} / \mathrm{deg}$ in these two different modes. Here, the sensitivity of the sensor $(\mathrm{S}=\Delta \mathrm{V} / \Delta \mathrm{P}$ or $\mathrm{S}=\Delta \mathrm{V} / \Delta \Theta$, where $\Delta \mathrm{P}$ or $\Delta \Theta$ are the relative number of changes in pressure and bending angle, $\Delta \mathrm{V}$ is the relative amount of change in the open-circuit voltage) was numerically equivalent to the linear slope of the curve. Stability of the sensor patches during (c) the pressing model and (d) the bending model, both of which exhibited long-term stability over 4000 dynamic loading cycles. 


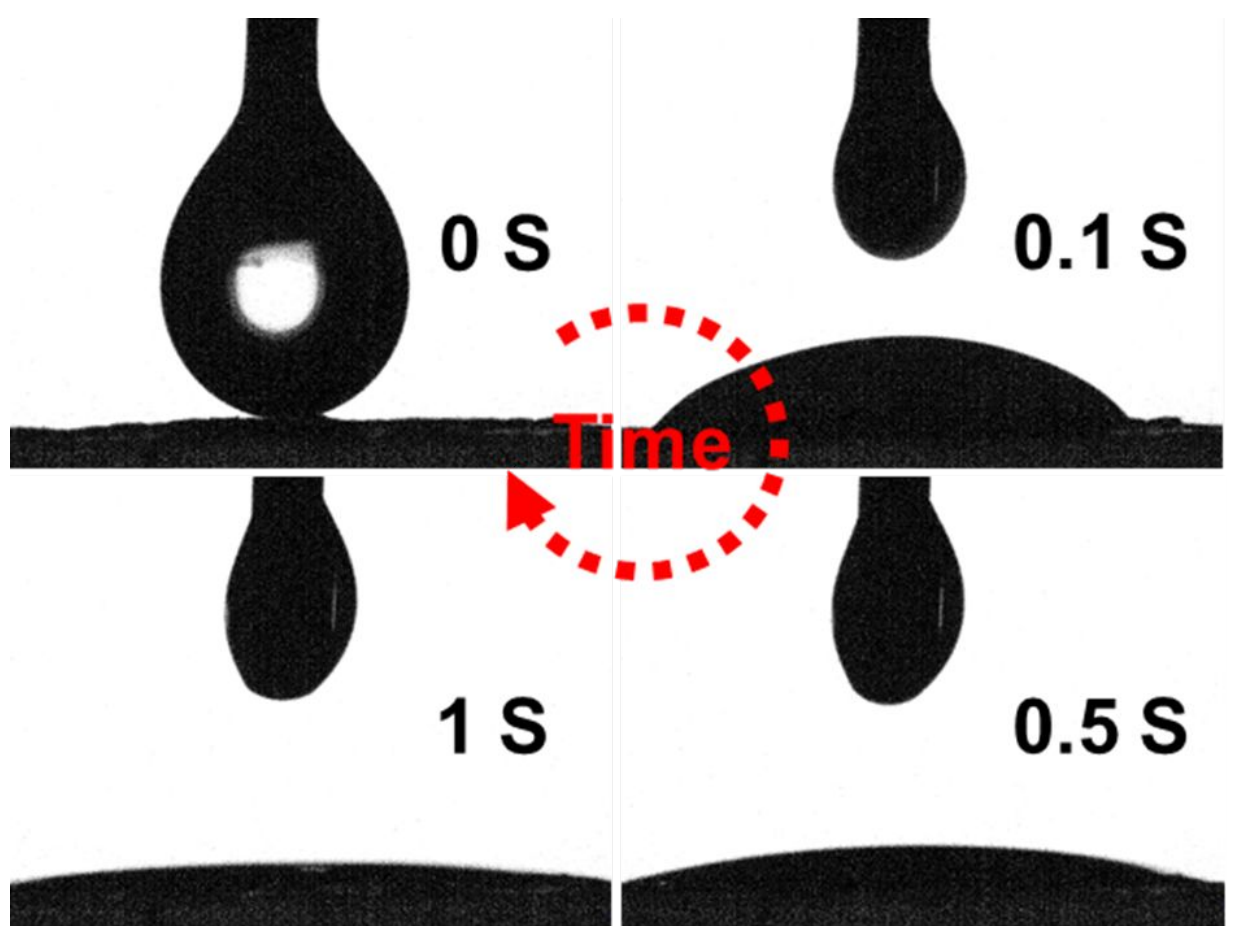

Figure S9. Electrolyte wettability of the BTO NF films. The BTO NF films had an excellent electrolyte penetration ability since it could completely absorb the liquid electrolytes in $1 \mathrm{~s}$, enabling a fast electrolyte wicking speed. 

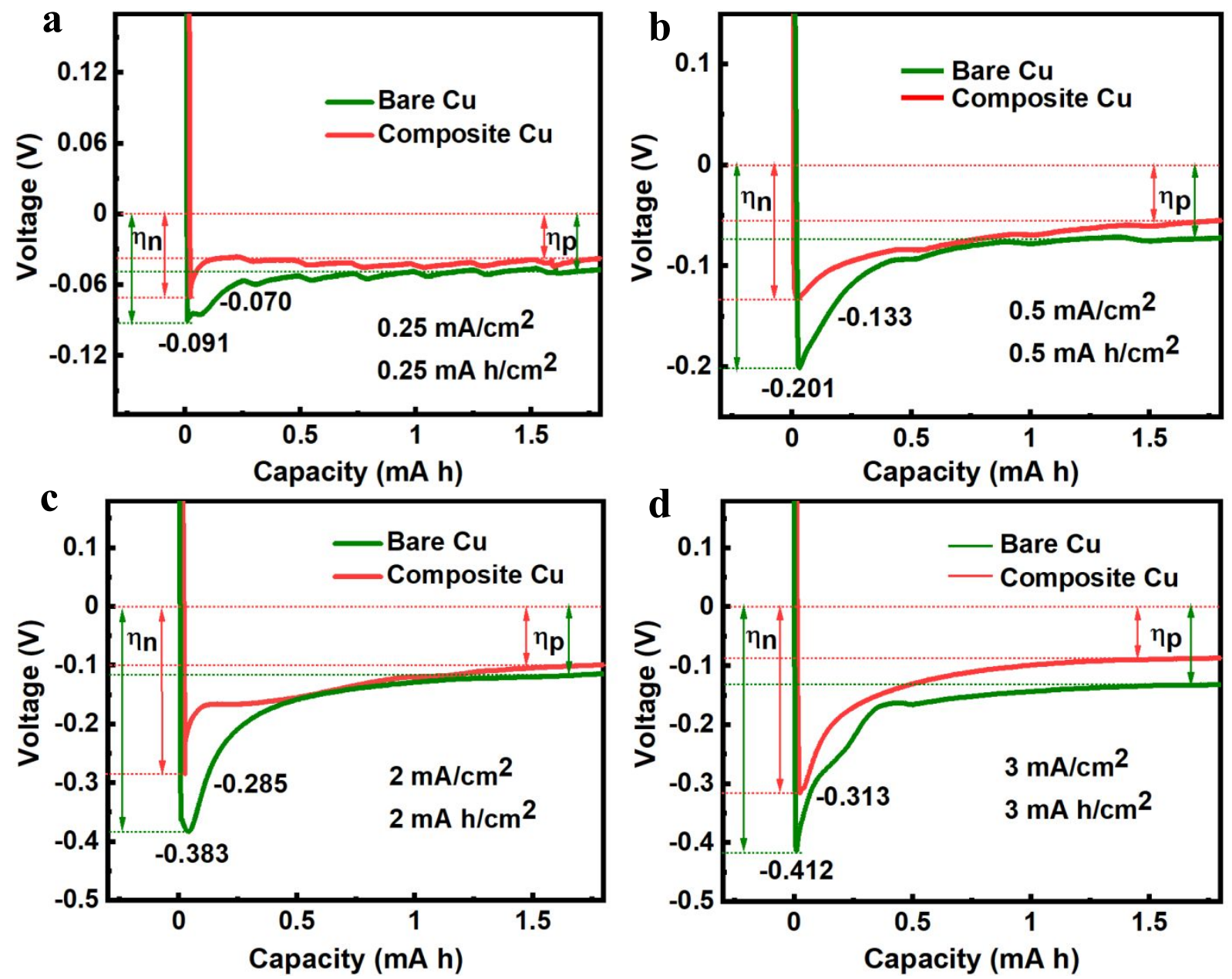

Figure S10. Nucleation overpotential curves of different $\mathrm{Li} \| \mathrm{Cu}$ cells at different current densities. The BTO NF film effectively reduced the overpotentials of Li-nucleation at different current densities, and thus decreased the nucleation resistances during Li-plating. 


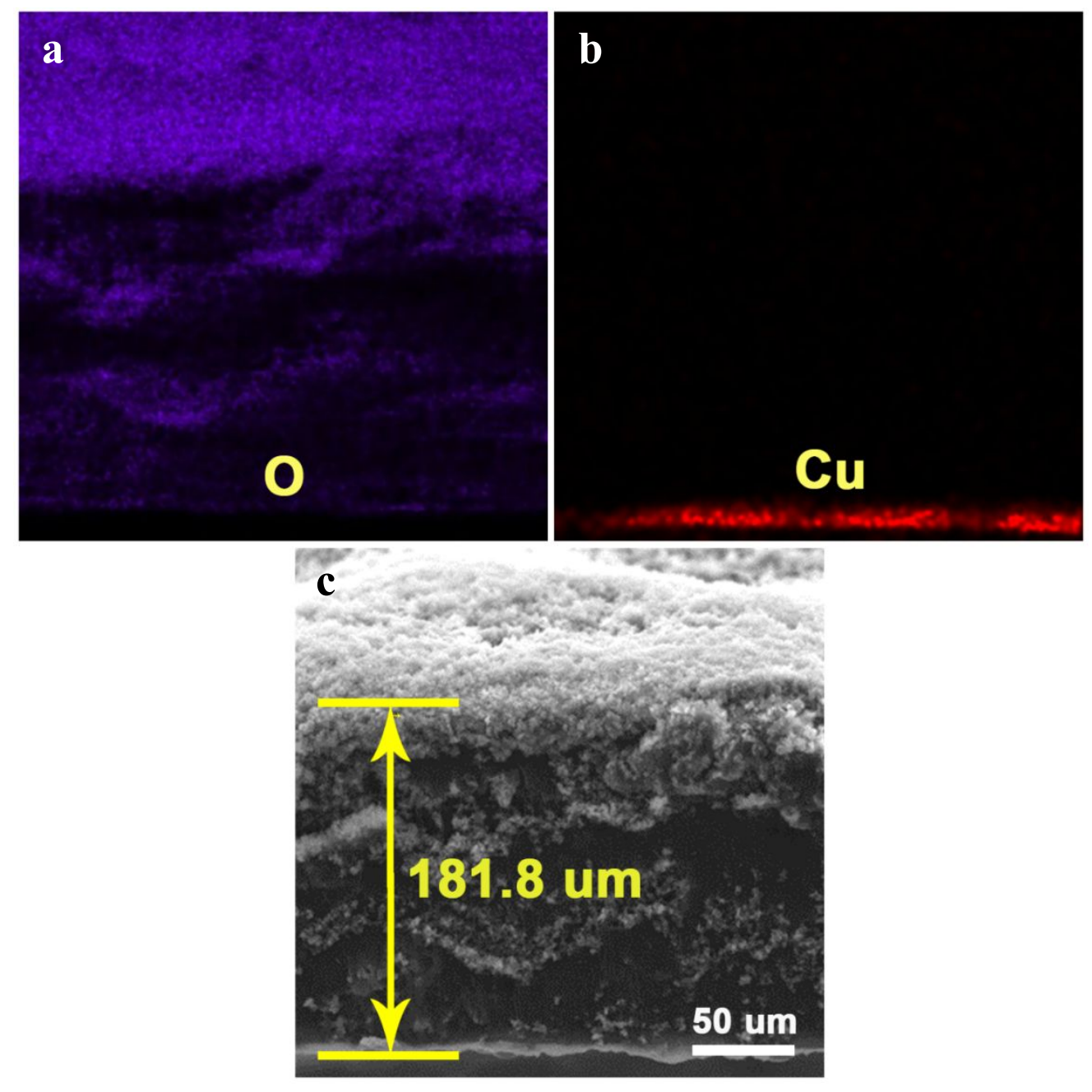

Figure S11. (a-b) The cross-sectional EDS mappings of (c) a bare Cu-electrode after cycling. The O-atoms were loosely distributed on the bare $\mathrm{Cu}$-electrode, indicating that a mossy-Li layer composed of dendrites was formed. The thickness of the mossy Li-layer was $181.8 \mu \mathrm{m}$. 

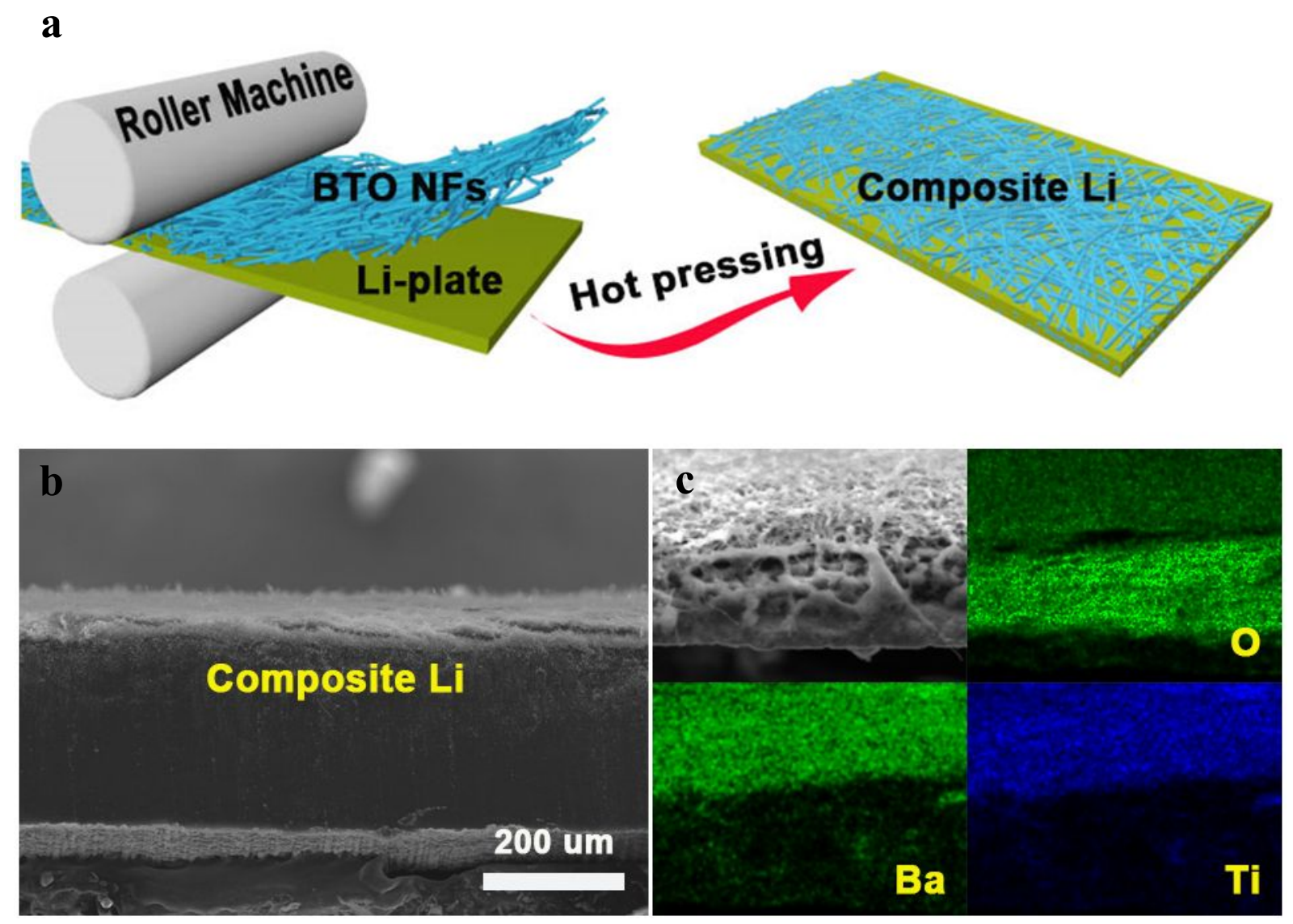

Figure S12. (a) A schematic diagram for preparing the integrated composite Li-anodes. The composite Li-anode was prepared by a facile hot-rolling method. Specifically, in the vacuum manual box, a BTO NF film and a Li sheet were first superimposed and sealed in an aluminum plastic film. Then, the BTO-Li composite anode was fabricated by hot-pressing at $180{ }^{\circ} \mathrm{C}$ for $180 \mathrm{~S}$ using a roller machine. (b) The cross-sectional image and (c) the corresponding EDS mappings of a composite Li-anode. The BTO NF film maintained well of its fibrous morphology in the composite anodes and was uniformly combined with the Li-metal. 

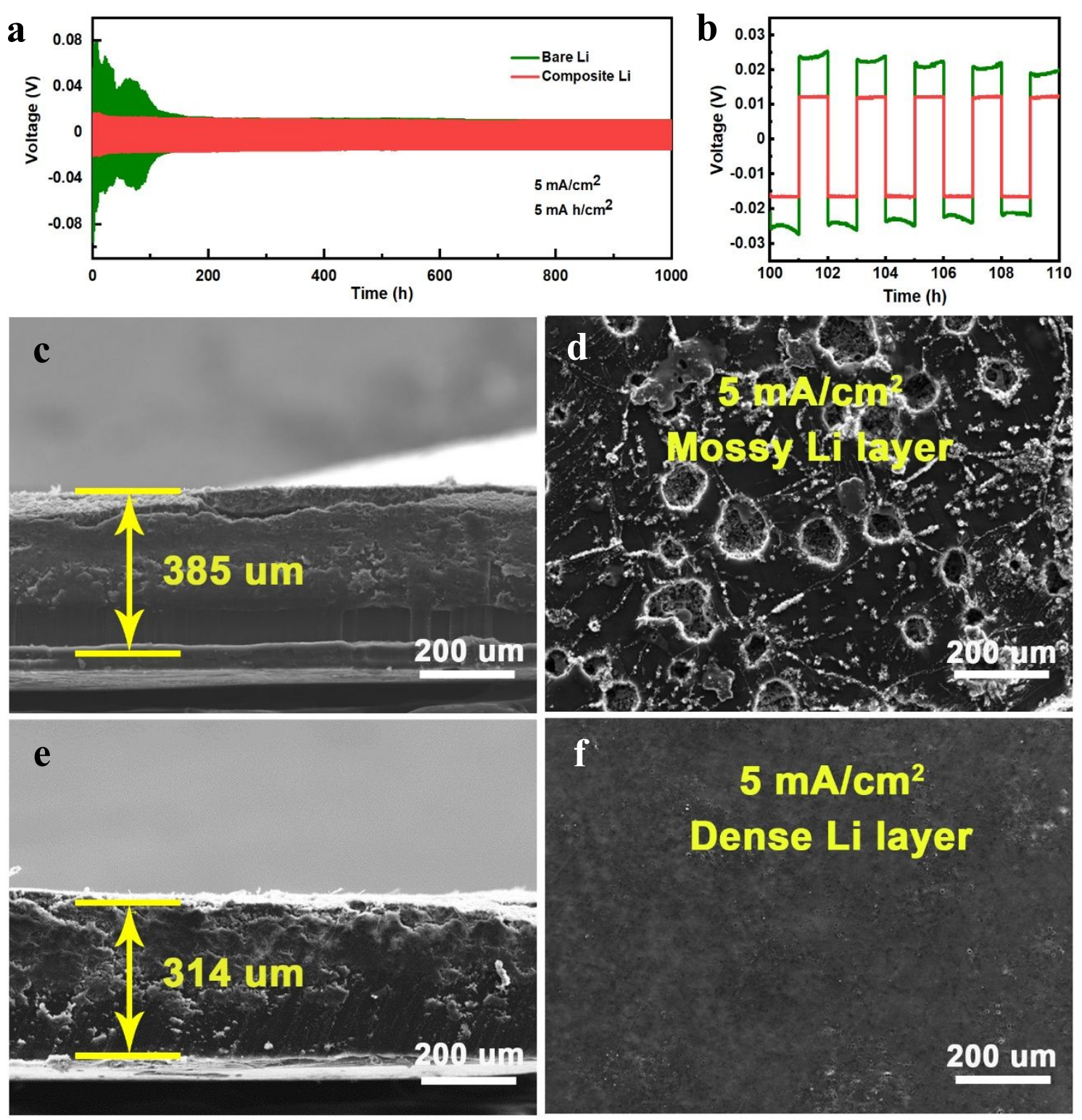

Figure S13. Investigation of Li-ion plating/stripping behaviors on Li-anodes. (a) Long

cycling stability test of $\mathrm{Li} \| \mathrm{Li}$ cells at $5 \mathrm{~mA} / \mathrm{cm}^{2}$. (b) Partially enlarged view of the galvanostatic charge/discharge curves. At $5 \mathrm{~mA} / \mathrm{cm}^{2}$, the composite Li-anode delivered a long cycle stability of over $1000 \mathrm{~h}$. However, the bare Li-anode showed an oscillated voltage profile and failed quickly. Morphology characterization of (c-d) the bare and (e-f) the composite Li-anodes after cycling at $5 \mathrm{~mA} / \mathrm{cm}^{2}$ over $100 \mathrm{~h}$. The bare Li-anode had an uneven surface, and the thickness of the Li metal was $385 \mu \mathrm{m}$. The composite $\mathrm{Li}$ anode had a flat and dense surface with a small thickness of $314 \mu \mathrm{m}$. Therefore, the BTO NF film could guide Li-plating and stripping to a more uniform distribution. 

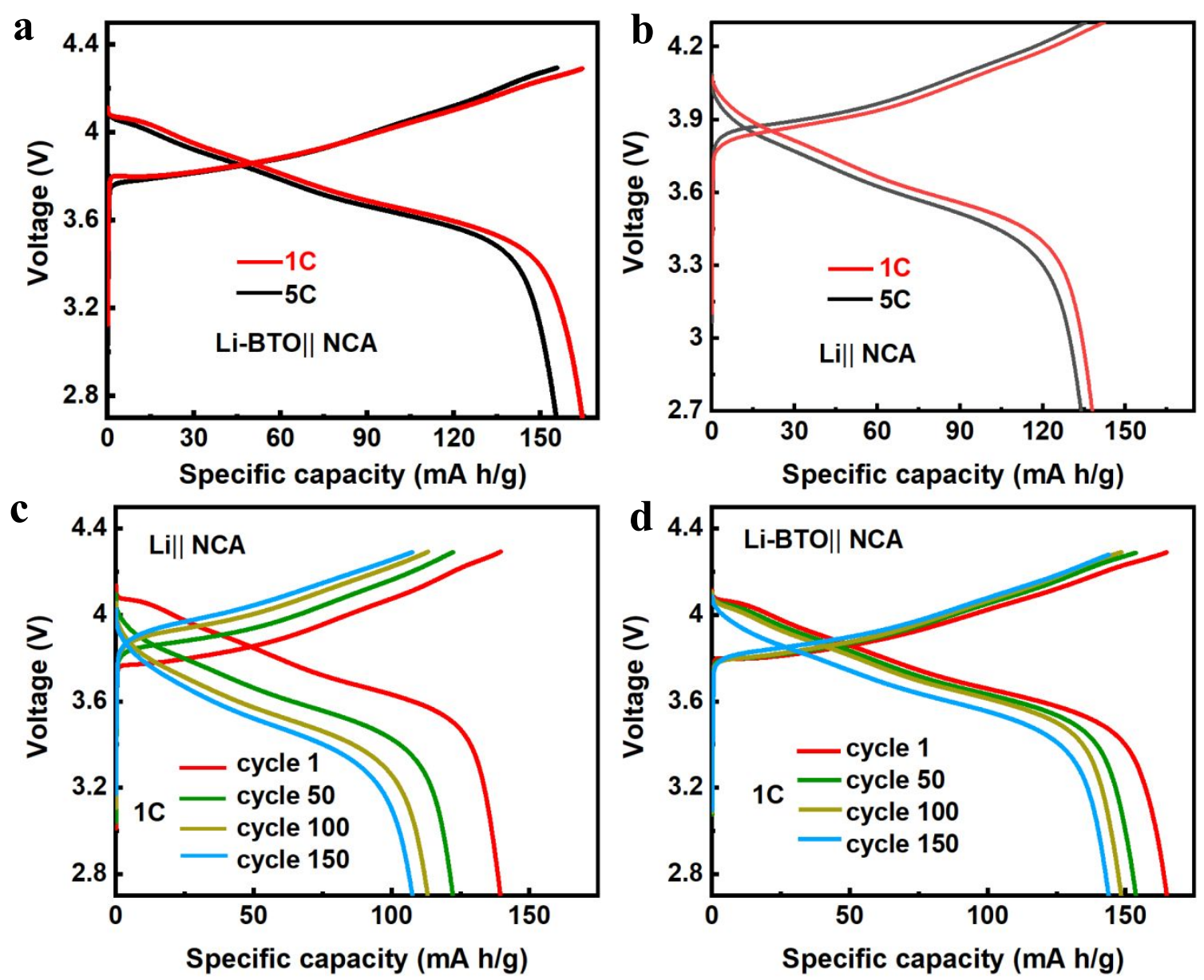

Figure S14. Charge/discharge curves of the Li//NCA and Li-BTO//NCA batteries. The first charge/discharge profile of (a) Li-BTO $\mid$ NCA and (b) Li $\mid$ NCA at $1 \mathrm{C}$ and $5 \mathrm{C}$. The initial discharge capacities of the $\mathrm{Li} / \mathrm{BTO} \| \mathrm{NCA}$ batteries at $1 \mathrm{C}$ and $5 \mathrm{C}$ were $164.5 \mathrm{~mA} \mathrm{~h} / \mathrm{g}$ and 155.1 $\mathrm{mA} \mathrm{h/g}$, respectively, which were both increased compared to $\mathrm{Li} \| \mathrm{NCA}$ batteries. Charge/discharge curves of (c) Li $\|$ NCA and (d) Li-BTO $\|$ NCA at $1 \mathrm{C}$ under different cycles. The Li $\|$ NCA batteries delivered a small initial discharge capacity of $139 \mathrm{~mA} \mathrm{~h} / \mathrm{g}$, which quickly decreased to $107 \mathrm{~mA} \mathrm{~h} / \mathrm{g}$ over 150 cycles with a low capacity retention of $76.9 \%$. In contrast, the Li-BTO||NCA batteries delivered a high initial discharge capacity of $164 \mathrm{~mA} \mathrm{~h} / \mathrm{g}$, which gradually decreased to $142 \mathrm{~mA} \mathrm{h/g}$ over 150 cycles with a high capacity retention of $87.6 \%$. 

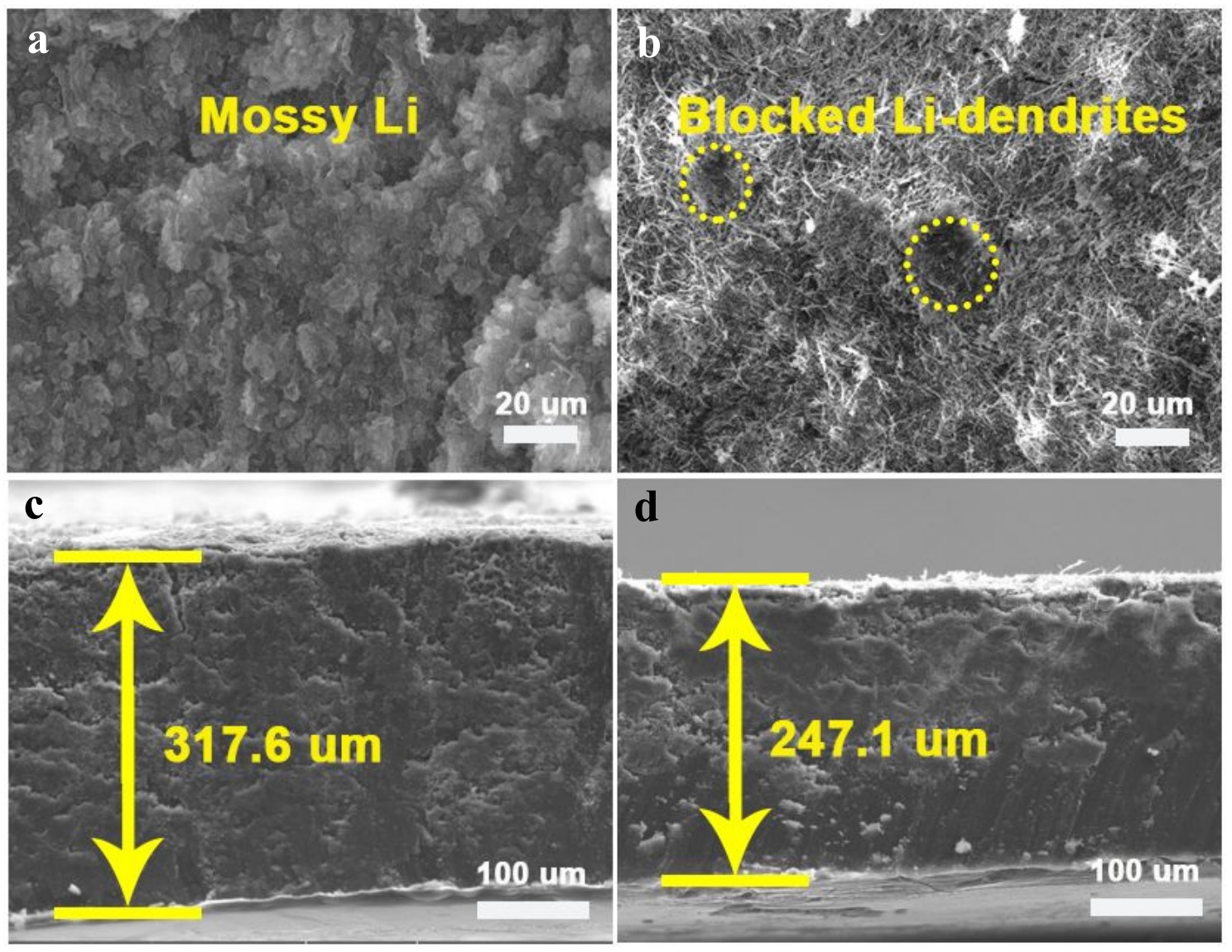

Figure S15. Morphology characterization of the Li//NCA and Li-BTO//NCA batteries at

5 C over 50 cycles. The surface and sectional morphology of anodes in (a, c) Li//NCA and (b, d) $\mathrm{Li} \mathrm{BTO//NCA}$ batteries that runned at $5 \mathrm{C}$ over 50 cycles. After 50 cycles, many vertically grown dendrites were observed on the bare Li-anode. On the contrary, blocked Li-dendrites could be observed after removing the BTO NF film from the composite anode. Moreover, the thickness of the composite anode $(247.1 \mu \mathrm{m})$ was much smaller than that of the bare Li-anode (317.6 $\mu \mathrm{m})$. These results confirmed the inhibitory effect of BTO NFs on dendrites. 